Beatriz H. Juarez*

\title{
The Role of Halogens in the Synthesis of Semiconductor Nanocrystals
}

\begin{abstract}
The presence of halogen compounds in the synthesis of semiconductor nanocrystals (NCs) produced by hot injection drastically influence their capping ligand sphere and/or chemical reactivity, allowing for the growth of hybrid systems, including composites combining NCs decorating carbon $\mathrm{sp}^{2}$ surfaces. As a result, the presence of halide anions on the surface of some NCs has proven to have a positive impact on the optical and electrical properties. In this work the effects that halogen co-solvents (including alkylchlorides, bromides, and iodides) induce in the synthesis of rod-like Wurtzite CdSe NCs generated by hot-injection will be reviewed. The proposed mechanism of the reaction as well as a detailed characterization of the capping ligand sphere by Nuclear Magnetic Resonance (NMR) and X-Ray photoelectron Spectroscopy (XPS) will be discussed. Correlated cyclic voltammetry (CV) and XPS studies have been performed to address the effect of the halide anions on the NCs surface. Finally an example of the effect of the addition of chlorine-containing co-solvents on the synthesis of $\mathrm{PbS}$ rock-salt NCs will be shown.
\end{abstract}

Keywords: Semiconductor Nanocrystals, Halide Anions, Chlorine Solvents, Shape Transformation, Hybrid Systems, Carbon Nanotubes-Semiconductor Nanocrystals Composites, NMR Ligand Sphere Characterization, XPS Studies, Electrochemistry of Nanocrystals.

DOI 10.1515/zpch-2014-0594

Received August 27, 2014; accepted November 4, 2014

Dedicated to Horst Weller on the occasion of his $60^{\text {th }}$ birthday

\footnotetext{
*Corresponding author: Beatriz H. Juarez, Applied Physical-Chemistry Department, Universidad Autónoma de Madrid, Francisco Tomás y Valiente 7, Campus Cantoblanco, Madrid 28049, Spain; and IMDEA Nanoscience, Faraday 9, 28049, Campus Cantoblanco, Madrid, Spain, e-mail: beatriz.hernandez@uam.es
} 


\section{Introduction}

The presence of halogen compounds in the synthesis of semiconductor nanocrystals (NCs) produced by hot injection [1] drastically influences their capping ligand sphere and/or their chemical reactivity [2-5], allowing for the growth of hybrid systems [6], including composites of NCs decorating carbon $\mathrm{sp}^{2}$ surfaces [7]. Several works have reported on chemical and photochemical processes taking place during the growth of semiconductor NCs in the presence of halide or acetate anions [8]. Changes in the capping ligand sphere triggered by halogen compounds may provoke transformations of the NCs shape [9-11]. There are examples of different materials, such as $\mathrm{MnS}$ and CdSe where hexagonal bullets, pyramidal, pencil, or diamond shapes are formed when chloride precursors are employed, which points to a general effect of chloride in wurtzite-type structures [9-11]. In the case of rock-salt PbS NCs produced by hot-injection, the addition of chlorinecontaining co-solvents triggers fusion of the NCs over certain crystallographic facets through oriented attachment $[12,13]$ transforming the initial dots into $2 \mathrm{D}$ sheets [14].

The presence of halide anions on the surface of some NCs produced by hotinjection has proven to have a positive impact on the optical and electrical properties [15]. Halide anions can be considered as the shortest X type (ionic) ligands, facilitating charge transport through NCs. The passivating role of halide anions in $\mathrm{PbS}$ systems has also been reported [16] and other reports have evidenced the passivating effect of chlorine on CdSe NCs, highlighting the possibility of using halogen molecules to efficiently displace both L- (coordinative) and/or X-type ligands $[17,18]$. Post-reaction treatments with chlorine-containing solutions seem to provide some benefits to PbSe colloidal NCs, such as more robust optical properties due to better passivation [19]. Furthermore, better performances of NC-based solar cells have been achieved upon chlorine-based treatments of the NCs followed by thermal annealing [20].

In this work, the effects that halogenated co-solvents (including alkylchlorides, bromides, and iodides) induce in the synthesis of rod-like wurtzite CdSe NCs [21] generated by hot-injection is reviewed. For example, rod-like CdSe NCs undergo a shape transformation to pyramidal shape in the presence of chlorinebased co-solvents, as schematically depicted in Figures 1a-1c. The proposed mechanism of the reaction as well as a detailed characterization of the capping ligand sphere of both, rod-like and pyramidal NCs by Nuclear Magnetic Resonance (NMR) and X-Ray Photoelectron Spectroscopy (XPS) will be discussed. Correlated Cyclic Voltammetry (CV) and XPS studies have been performed to address differences between the NCs surfaces. 

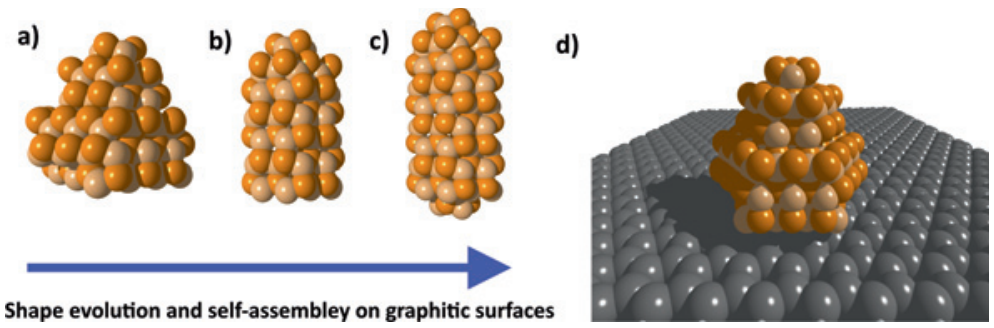

Figure 1: $(a-c)$ Sketch of the shape evolution undergone by rod-like CdSe NCs into pyramids in the presence of halogen co-solvents and d) tendency to attach to carbon $\mathrm{sp}^{2}$ lattices such as those of carbon nanotubes, highly oriented pyrolytic graphite (HOPG), or flakes of a few layers (see text).

These studies allow identifying chemical environments and surface site modifications, which are essential to understand the stability and performance of NCs acting as active layers in optoelectronic devices. Furthermore, it has been proven that the chlorine-modified ligand sphere triggers self-assembly of a single monolayer of CdSe NCs on graphitic surfaces (see Figure 1d) such as carbon nanotubes or highly oriented pyrolytic graphite (HOPG), either as bulk or in the form of a few layers of graphene. This opens room for semiconductor NCs-graphene composites for applications in catalysis, photovoltaics or energy storage [22]. Finally an example of the effect of the addition of chlorine-containing co-solvents on the synthesis of $\mathrm{PbS}$ rock-salt NCs will be shown as well [14].

\section{Characterization of the capping ligand sphere of rod-like and pyramidal CdSe NCs}

As mentioned, rod-like CdSe NCs undergo a shape transformation to pyramidal shape ones in the presence chlorine-containing co-solvents, such as 1,2dichloroethane (DCE). The synthesis includes the addition of DCE to a mixture of cadmium oxide $\mathrm{CdO}$ and octadecylphosponic acid ODPA prior to the addition of selenium Se dissolved in trioctylphosphine TOP. We found that there is a double role of DCE depending on the $\mathrm{Cd} / \mathrm{Se}$ precursor molar ratio (either under excess of $\mathrm{Cd}$ or Se precursors) [23]. While NCs produced under conditions with an excess of $\mathrm{Cd}$ precursor show rod-like shape and a comparatively low amount of $\mathrm{Cl}$ on their surface, NCs produced under conditions with an excess of Se precursor modify their shape from rods to pyramids and contain higher amounts of the halogen on their surface. In order to obtain quantitative information of the surface composition and to investigate the integration of $\mathrm{Cl}$ in the ligand NCs shell, XPS analyses 

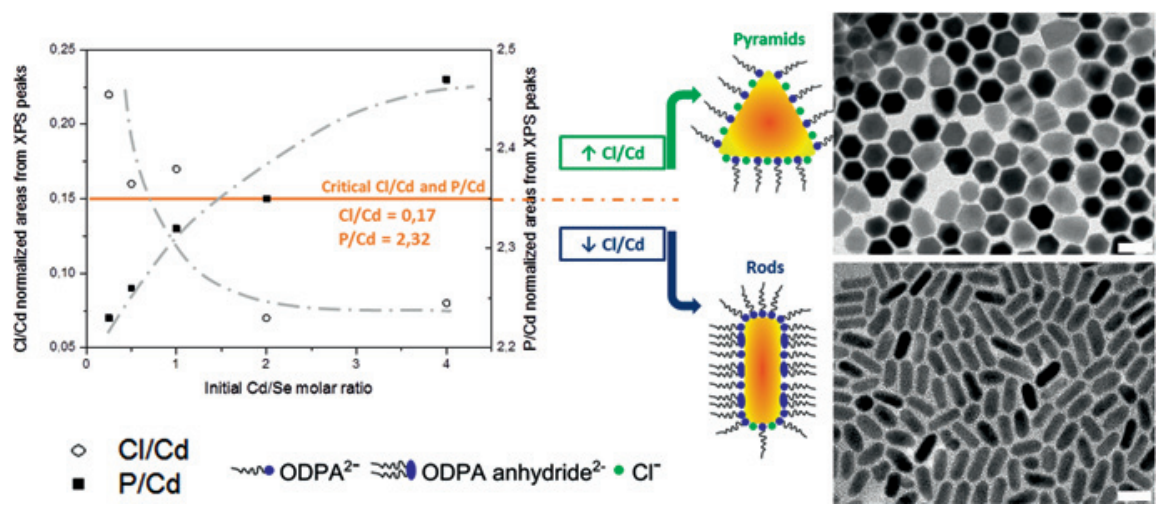

- $\mathrm{Cl} / \mathrm{Cd}$

- P/Cd

$m=\mathrm{ODPA}^{2-}=$ ODPA anhydride $e^{2 \cdot} \cdot \mathrm{Cl}^{-}$

Figure 2: $\mathrm{Cl} / \mathrm{Cd}$ (circles) and $\mathrm{P} / \mathrm{Cd}$ (dark squares) normalized areas calculated from XPS data and plotted versus the $\mathrm{Cd} / \mathrm{Se}$ precursor molar ratios used in the synthesis (CdO and TOP@Se). The horizontal continuous line indicates the minimum $\mathrm{Cl}$ value to promote the re-shaping of rod-like to pyramidal-like NCs. The discontinuous curved lines are only a guide for the eyes. The experimental error associated with the relative peak areas is $10 \%$. The drawings represent pyramids and rods capped with ODPA-related ligands and chloride. TEM images on the right correspond to both types of NCs. Scale bars $20 \mathrm{~nm}$.

have been performed. Figure 2 shows the XPS $\mathrm{Cl} / \mathrm{Cd}$ and $\mathrm{P} / \mathrm{Cd}$ normalized areas plotted as a function of the $\mathrm{Cd} / \mathrm{Se}$ precursor molar ratio in the synthesis. Since the $\mathrm{P} 2 \mathrm{p}$ and $\mathrm{Cl} 2 \mathrm{p}$ XPS peaks are related exclusively to the NCs ligand shell, the normalized areas $\mathrm{Cl} / \mathrm{Cd}$ and $\mathrm{P} / \mathrm{Cd}$ between elements comprising the ligand shell $(\mathrm{Cl}$ or $\mathrm{P})$ and a component of the $\mathrm{NC}$ core ( $\mathrm{Cd}$ in this case) were calculated. These ratios do not correspond to quantitative concentration values of elements present in the samples but to qualitative amounts of different elements composing the shell relative to a given amount of $\mathrm{Cd}$. As shown in Figure 2, for low $\mathrm{Cd} / \mathrm{Se}$ values $(\mathrm{Cd} / \mathrm{Se}<1)$, low $\mathrm{P} / \mathrm{Cd}$ and high $\mathrm{Cl} / \mathrm{Cd}$ values are observed. This trend is inverse for high $\mathrm{Cd} / \mathrm{Se}$ values $(\mathrm{Cd} / \mathrm{Se}>1)$. The fact that the $\mathrm{Cl} / \mathrm{Cd}$ and $\mathrm{P} / \mathrm{Cd}$ ratios follow opposite trends as a function of the $\mathrm{Cd} / \mathrm{Se}$ precursor molar ratio supports a mechanism where the adsorption of chlorine on the NCs surface entails the substitution of ODPA-related ligands (containing P), and triggers re-shaping of the initial rods. The horizontal line in Figure 2 corresponds to the minimum $\mathrm{NC}$ chlorine content (as $\mathrm{Cl} / \mathrm{Cd}$ ) necessary to perform the re-shaping from rods to pyramids (around 0.17). The drawings represent pyramids and rods capped with ODPA-related ligands and chloride. TEM images on the right correspond to both types of NCs. In order to investigate the chemical changes on the P sites, comparative studies have been carried out on samples synthesized in the absence of chlorinated co-solvents (rod-like NCs) and samples showing a high concentration of chlorine on the surface (pyramidal NCs) by solid NMR and synchrotron XPS. 

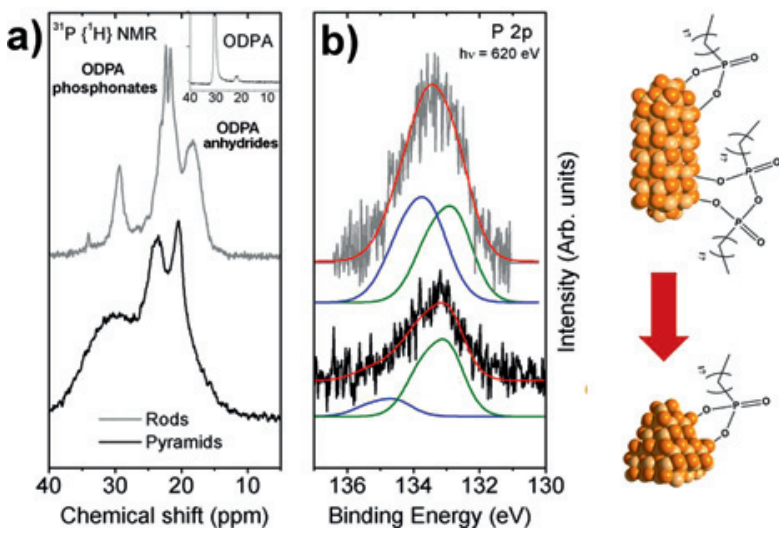

Figure 3: Solid ${ }^{31} \mathrm{P}\left\{{ }^{1} \mathrm{H}\right\}$ NMR of rod-like (upper gray line) and pyramidal CdSe NCs (lower black line). Inset: pure ODPA. (b) P $2 p$ region acquired for rod-like (upper gray line) and pyramidal NCs (lower black line) by XPS ( $h v=620 \mathrm{eV}$ ). The P $2 p$ peaks can be deconvoluted in two components with different binding energies (green and blue lines for $\mathrm{ODPA}^{2}$ - and anhydrides, respectively). A sketch indicating rod-like NCs capped with both ODPA ${ }^{2}$ - and anhydrides and pyramidal NCS capped exclusively with $\mathrm{ODPA}^{2}$ - is also represented.

Figure 3a shows the solid ${ }^{31} \mathrm{P}\left\{{ }^{1} \mathrm{H}\right\}$ NMR spectra for rod-like CdSe NCs (upper grey line) and pyramids (lower black line), respectively. The spectra show sets of well-defined peaks. The comparison reveals that the transformation involves a shift and/or broadening of the signals, indicating a different chemical environment for P. The ${ }^{31} \mathrm{P}\left\{{ }^{1} \mathrm{H}\right\}$ NMR spectrum of rod-like NCs (Figure 3a, upper line) shows a peak at $18.3 \mathrm{ppm}$; a prominent signal around $22-23 \mathrm{ppm}$ terminated in two narrow peaks at 21.7 and $22.4 \mathrm{ppm}$; and two additional signals at 29.3 and $34.0 \mathrm{ppm}$. The absence of peaks at higher chemical shifts (>35 ppm) discards the presence of TOPO bonded to the NC surface, in agreement with previous studies [21]. Three of those signals (29.3, 22.4, and $21.7 \mathrm{ppm}$ ) can also be found in the solid ${ }^{31} \mathrm{P}\left\{{ }^{1} \mathrm{H}\right\}$ NMR spectrum of pure ODPA (see inset). The dominating contribution at $30.4 \mathrm{ppm}$ is similar to the value reported for solution $\mathrm{ODPA}^{31} \mathrm{P}$ NMR [21, 24]. The signals observed in Figure 3a at 22 and $18.3 \mathrm{ppm}$ can be assigned to ODPA species directly bound to $\mathrm{Cd}$ in the form of octadecylphosphonates (double deprotonated $\mathrm{ODPA}^{2-}$ ) and anhydrides of phosphonic acids, respectively [2528]. The presence of ODPA (neutral molecule) could be related to the hydrolysis of anhydrides, and hydrogen bonding between ODPA molecules might be the origin of the peaks at 22.4 and $21.7 \mathrm{ppm}$, according to previous work [24]. Finally, we attribute the small signal at 34 ppm to a small contribution of Se@TOP. Thus, out of all the contributions found in the solid ${ }^{31} \mathrm{P}$ NMR spectra, the two main components that can be unambiguously attributed to species in the ligand shell are those 
corresponding to ODPA ${ }^{2-}$ and ODPA anhydrides. For pyramidal NCs (Figure 3a, lower line), the peaks previously assigned to ODPA ${ }^{2-}$ and ODPA anhydrides are slightly shifted downfield when compared to the spectrum of rod-like NCs. Furthermore, a slight downfield shift is also observed by solid state ${ }^{1} \mathrm{H}$ MAS NMR experiments in the pyramidal NCs compared to rod-like (not shown) [29]. The shift in the whole spectrum could be attributed to the influence of adjacent chlorine atoms in the capping shell. Alternatively, this shift may be related to a slightly different binding against the predominant (1-101) polar facets terminated in $\mathrm{Cd}$ in the case of pyramids $[7,30]$ compared to the predominant non-polar (01-10) facets in rods. The range between 25 and $40 \mathrm{ppm}$ shows a broad undefined peak, and the two previous contributions at 21.7 and $22.4 \mathrm{ppm}$ are not observed, which may indicate breaking of the hydrogen bonds in the presence of chlorine. Since the ${ }^{31} \mathrm{P}$ NMR signals of phosphonic acid chlorides are shifted 14 to $20 \mathrm{ppm}$ downfield from the corresponding phosphonic acids [31] a P-Cl bound is discarded. Changes in the $\mathrm{P}$ core levels of the NCs are also apparent in synchrotron XPS. Figure $3 \mathrm{~b}$ shows the $\mathrm{P} 2 \mathrm{p}$ region acquired for rod-like and pyramidal NCs, respectively. The natural line shape for $2 p$ levels is that of an asymmetric spin-split doublet, very different from the line shape found for rod-like NCs where two components with similar intensities, but different binding energies (132.92 and $133.74 \mathrm{eV}$, respectively) fit bests (spectrum in Figure 3b upper line). The shape of the XPS signal for pyramidal NCs (3b lower line) is, however, much more asymmetric and, thus, more consistent with a single environment of $\mathrm{P}$ on their surface. If two components are still allowed for fitting the experimental data, it is found that the intensity of the highest-binding energy one is now much smaller and both components have been shifted to higher binding energies (133.11 and $134.71 \mathrm{eV}$ respectively for the spectrum in Figure 3b, lower line). This small shift of about $0.1 \mathrm{eV}$ for both components follows the same trend of the downfield-shifted signals observed in the NMR spectrum upon chlorine incorporation. Furthermore, the width of the $\mathrm{P} 2 \mathrm{p}$ signal for anhydrides is slightly larger $(1.24 \mathrm{eV})$ than that related to the ODPA ${ }^{2-}$ molecules $(1.11 \mathrm{eV})$, which may be related to the variable phosphorus environment in anhydrides of different chain length. The fact that two main components are observed for the phosphorus signals related to ligand shell species both in XPS and NMR suggests identification between them. We attribute the signal at the lowest binding energy to ODPA ${ }^{2-}$ in agreement with previous results [26] and the signal at higher binding energy to anhydrides of ODPA. A sketch indicating rod-like NCs capped with both $\mathrm{ODPA}^{2-}$ and anhydrides and pyramidal NCs capped exclusively with $\mathrm{ODPA}^{2-}$ is also represented. 


\section{Proposed mechanism triggering the shape transformation}

According to the above information, the incorporation of $\mathrm{Cl}$ as ligand seems to entail the substitution of ODPA-related ligands (containing P) and further reshaping of the initial rods. After the characterization shown in Figure 3 it is reasonable to say that the addition of a chlorine-containing co-solvent to the reaction media of CdSe rod-like NCs leads to the incorporation of chlorine by selective substitution of anhydrides of octadecylphosphonic acid, while octadecylphosphonates $\left(\mathrm{ODPA}^{2-}\right)$ remain anchored to the NCs surface. Furthermore, as previously mentioned, the shape transformation occurs exclusively under high Se precursor concentrations (Se@TOP). In order to understand the role of the halogen-containing co-solvent in the reaction a series of blank experiments were performed (not shown) [23] to conclude that the reaction between TOP (or TOP@Se) and DCE is responsible for the incorporation of $\mathrm{Cl}$ into the NCs ligand shell, triggering the re-shaping of the NCs. In order to follow the reaction and to understand the influence of this combination during the NCs synthesis, Nuclear Magnetic Resonance $\left({ }^{1} \mathrm{H}\right.$ NMR) and Mass Spectrometry (ESITOF) have been employed to analyze the reactivity between TOP and DCE at RT at $265^{\circ} \mathrm{C}$, the reaction temperature [23]. The ESI-TOF spectrum of TOP (Figure 4a) evidences the absence of significant impurities, indicating that the main reaction products should proceed from the reaction between TOP and DCE. Figure $4 \mathrm{~b}$ shows the ESI-TOF spectrum of the reaction product. The main peak dominating the spectrum is situated at $\mathrm{m} / \mathrm{z} 384.39$. This peak can be unambiguously attributed to ethane-1,2-diylbis(trioctylphosphonium)dichloride. This as-
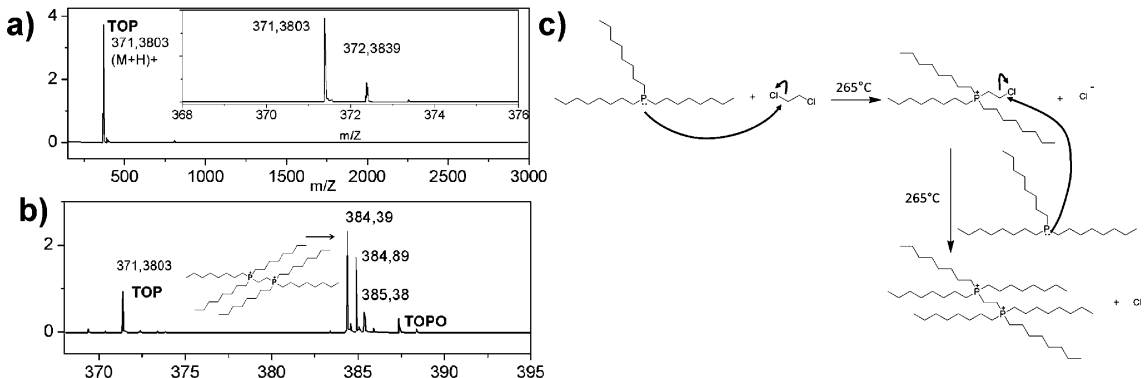

Figure 4: a) ESI-TOF mass spectrum of TOP, demonstrating no relevant impurities. The inset shows a smaller spectral range where the $(M+1)^{+}$and its corresponding isotopes are observed. b) ESI-TOF mass spectrum of the product obtained from the reaction between DCE and TOP at $265^{\circ} \mathrm{C}$, (ethane-1,2-diylbis(trioctylphosphonium)dichloride). c) Proposed mechanism for the chloride anions generation as products of the reaction between DCE and TOP at $265^{\circ} \mathrm{C}$. 
signment is also supported by the fact that the $M+1$ and $M+2$ peaks of ethane-1,2diylbis(trioctylphosphonium)dichloride are separated by a difference of 0.5 , according to a doubly charged product. The intensity relations correspond to the abundance of the carbon isotopes. The peaks at 371.38 and 387.37 are assigned to TOP and TOPO, respectively, and correspond to the singly charged species which have peaks of $M+1$ and $M+2$ separated by a difference of 1 . The presence of unreacted TOP and a small amount of TOPO is also supported by ${ }^{1} \mathrm{H}$ NMR (not shown) [23]. The peak in the ESI-TOF spectrum ( $\mathrm{m} / \mathrm{z} 1 / 4$ 384.39, Figure 4b), together with the disappearance of the DCE peaks in ${ }^{1} \mathrm{H}$ NMR after mixing with TOP at high temperature reported in [23] evidences the ethane-1,2-diylbis(trioctylphosphonium)dichloride generation. This reaction may occur through a substitutional mechanism, as depicted in Figure 4c. Thus, chloride anions are released in situ upon reaction between TOP and DCE at $265^{\circ} \mathrm{C}$ and are incorporated as ligands in the capping shell.

\section{Studies of other halogenated compounds}

In a previous work it was shown that depending on the chemical structure of the halogen molecule added to the reaction, the kinetic growth and the final particle shape vary [32]. Since chloride anions are responsible for the shape transformation from rods to pyramids, this evolution will take place faster at higher chloride concentration (within a certain range of concentrations). This was observed when comparing the evolution of different primary, secondary or tertiary haloalkanes, as reported in detail in [32]. Furthermore, the use of other compounds such as $\mathrm{HCl}$ [33] or other halogen-containing co-solvents releasing bromide or iodine anions were also tested [32]. In contrast to chloroalkanes, modifying the shape of the rods at relatively long reaction times (hours) during the ripening regime, the presence of bromoalkanes and especially iodoalkanes influences the reaction especially during the first stages, during the nucleation and the first stages of growth, producing rods with clear zig-zag terminated surfaces, as a result of the competition between ODPA and the halide anion. The larger influence of iodide and bromide during the first stages of growth (within a concentration range) is related with their stronger nucleophilic character with respect to chloride. In the reaction with TOP, iodide is a very good leaving group, followed by bromide and chloride. This means that iodide anions will be available in higher concentrations from the beginning of the reaction on, influencing the nucleation and growth to a higher extend than chloride anions, influencing the reaction during the ripening stage at longer reaction times. 

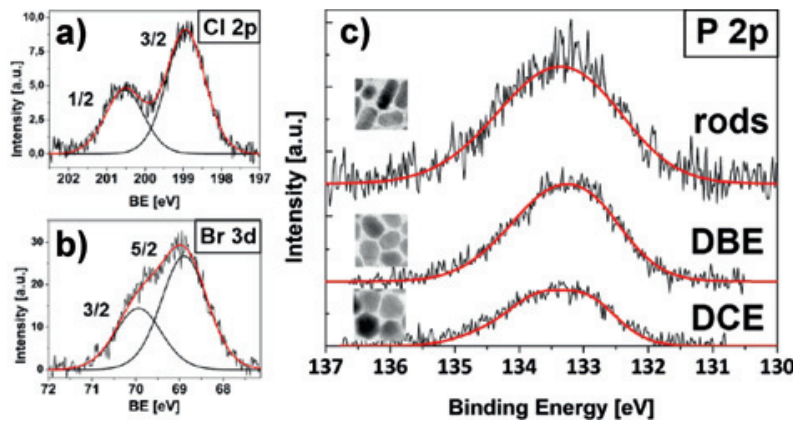

Figure 5: Synchrotron XPS data of the regions of $\mathrm{Cl} 2 \mathrm{p}$ (peak position 3/2:198.8 eV, (a)) and $\mathrm{Br}$ $3 \mathrm{~d}$ (peak position 5/2:68.9 eV, (b)) binding energies obtained at a photon energy of $620 \mathrm{eV}$. The areas of the P signals in (c) are normalized to the respective Cd $3 \mathrm{~d}$ signal to derive the atomic ratios. The ratio of the area between the $\mathrm{P} 2 \mathrm{p}$ and $\mathrm{Cd}$ signals (not shown) decreases from rods (0.14) to NCs produced with DBE (0.09) and DCE (0.05). The insets show TEM images of rod NCs produced in the absence of halogen co-solvents, pyramidal NCs with rounded facets when NCs are produced in the presence of DBE and pyramidal NCs with clear sharp facets in the presence of DCE.

To verify changes in the ligand shell produced by the different haloalkanes, synchrotron XPS data of rod-like and pyramidal NCs prepared with DCE and dibromoethane, DBE, have been compared in Figure 5. NCs produced in the presence of DCE show a clear $\mathrm{Cl}$ peak (Figure 5a) centered at $198.8 \mathrm{eV}$, an intermediate binding energy between the position of the $\mathrm{Cl} 2 \mathrm{p} 3 / 2$ in $\mathrm{CdCl}_{2}(198.4 \mathrm{eV})$ and that of $\mathrm{Cl} 2 \mathrm{p} 3 / 2$ in the more ionic $\mathrm{ZnCl}_{2}(199.1 \mathrm{eV})$. This position might be caused by an ionic interaction between chloride and the surface of NCs. The presence of the $\mathrm{Br}$ $3 d$ signal in the NCs produced with DBE (Figure $5 b$ ) suggests that $\mathrm{Br}$ from DBE reacts similarly to $\mathrm{Cl}$. Both $\mathrm{Cl}$ and $\mathrm{Br}$ signals turn out to be comparatively broad (fwhm Cl: $1.2 \mathrm{eV}, \mathrm{Br}: 1.3 \mathrm{eV}$ ) with respect to the Au signal reference $(0.7 \mathrm{eV}$ for the fwhm of the Au $4 \mathrm{f}$ peak), which indicates that these elements can bind indistinctly to several facets. From the XPS spectra of Figure 5c (P 2p), quantitative analysis of the surface-attached elements $\mathrm{P}$ and $\mathrm{Cl}$ (or $\mathrm{Br}$ ) shows that, for $\mathrm{Cl}$ pyramids, the ligand shell is composed of $\mathrm{Cl}$ species by about $(43 \pm 3) \%$, and the ligand shell of the $\mathrm{Br}$ pyramids contains $(31 \pm 5) \%$ of $\mathrm{Br}$. TEM images in Figure 5c (insets) show that the NCs with $\mathrm{Br}$ are less sharply faceted than those with $\mathrm{Cl}$ at the same reaction time (see Figure 1b), what can be understood when taking into account the already mentioned nucleophilic character, what in fact yields stronger $\mathrm{Cd}-\mathrm{Cl}$ bonds compared to $\mathrm{Cd}-\mathrm{Br}$ and $\mathrm{Cd}$-I. Thus, the presence of chloride strongly influences the ripening regime compared to bromide and iodide, showing an attenuated effect during this period. From these results we can conclude that the release rate of halide anions and their availability during nucleation and/or growth play a major role in the shape evolution. 
The shape transformation process can be understood as an etching process starting on the poorly passivated (000-1) Se terminated facet of wurtzite CdSe rods. In fact, the transformation starts exclusively from that tip, as ascertained as by TEM tomography [30]. The presence of water should be taken into account due to the fact that water, as byproduct of the Cd complexation with ODPA, favors the protonation of tightly bound phosphonate ligands, decreasing significantly the adsorption energy, according to density functional theory (DFT) calculations [32]. In this scenario, diffusion of halide anions (in particular, chloride) may compete with ODPA-based ligands. As also confirmed by DFT, ODPA anhydrides are unstable on the sloped (10-1-1) facet, whereas all halide ions show relatively high adsorption energy values for this facet [32]. Thus, on the basis of these results, the co-adsorption and stabilization of phosphonates and chloride anions on the sloped facet explains the pyramidal shape.

\section{Electrochemical studies of rod-like and pyramidal NCs}

Cyclic Voltammetry (CV) is a technique widely used to study semiconducting colloids [34-37], including many studies to match the optical and electrochemical gaps [38, 39]. To address the effect of chloride forming part of the ligand shell, the CV characterization of rods and pyramids was performed. Different voltammetric responses have been recorded depending on the scan potential direction and capping ligand shell composition, as reported in detail in [40]. Correlated CV and XPS measurements allow both to observe the difference in the electrochemical response of differently capped NCs and to identify chemical processes that Cd and Se surface atoms undergo under electrochemical treatments [40]. The combination of these techniques might be very useful to identify undesired effects on NCs-based devices affected by non-optimized charge injection/separation of charges. Figure 6 shows the voltammetric response of pyramids (red line) and rods (green line) when the initial scan direction is anodic $(0$ to $+2 \mathrm{~V})$ followed by a cathodic scan $(+2 \mathrm{~V},-2 \mathrm{~V})$. It is important to notice that upon a first cathodic scan (from 0 to $-2 \mathrm{~V}$, not shown) [40] no reduction peaks are recorded regardless of size, shape, or concentration of NCs on the electrode. On the contrary, in the subsequent anodic scan to $+2 \mathrm{~V}$, clear oxidation processes can be recorded in the voltammogram for all samples. These results are in agreement with an unfavorable cathodic reduction and a favorable anodic oxidation of CdSe at room temperature [41-43]. A second cathodic scan to $-2 \mathrm{~V}$ after previous oxidation is characterized by a reduction peak centered at $-1.4 \mathrm{~V}$. In all cases, since the reduction 


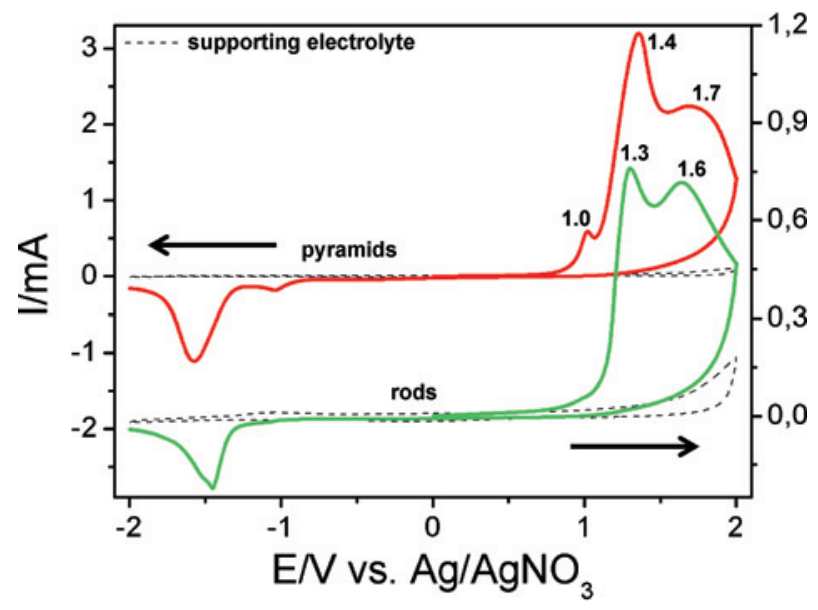

Figure 6: Cyclic voltammetry of pyramidal shape and rod-like NCs where the potential was initially scanned in the anodic direction. The complete cycle includes $(0,+2 \mathrm{~V},-2 \mathrm{~V})$. Electrolyte solution: $0.1 \mathrm{M}$ TBAP in acetonitrile; $v=0.1 \mathrm{~V} \mathrm{~s}^{-1}$.

peaks are only recorded upon previous anodic oxidation the potential difference between the anodic and cathodic responses cannot be assigned to the energetic positions of valence and conduction bands. According to our studies, the reduction peaks are assigned to the reduction of previously oxidized species on the surface of the NCs and will be further characterized in detail by XPS (more details can be found in [40]). From the voltammograms shown in Figure 6 two remarkable facts can be extracted: (i) the potential difference between the anodic and cathodic processes is about $2 \mathrm{~V}$ in both cases and (ii) the anodic area, obtained by subtraction of the background current, is higher than the area corresponding to the cathodic processes. The voltammogram for pyramidal NCs is composed of two defined peaks at 1.4 and $1.7 \mathrm{~V}(1.3$ and $1.6 \mathrm{~V}$ in rods) and a small peak centered at $1 \mathrm{~V}$, which cannot be found in the case of rod-like NCs. The two observable peaks separated $0.3-0.4 \mathrm{~V}$ in the anodic scans both for rods and pyramids might be related to the oxidation of two different atomic chemical environments. This potential difference is about $57 \mathrm{KJ} \mathrm{mol}^{-1}$ for the electronic transference of $n=2 \mathrm{e}^{-}$. Therefore, these peaks may correspond to the oxidation of Se located in different NCs facets for both rods and pyramids. Alternatively, these peaks can be assigned to different oxidation states of Se [44]. Several differences were found depending on the initial scanning direction and reported in [40].

Correlated XPS measurements have been employed to characterize the different NCs surfaces and to understand the changes produced during the oxidation and reduction processes (Figure 7). By XPS three different oxidation processes for 

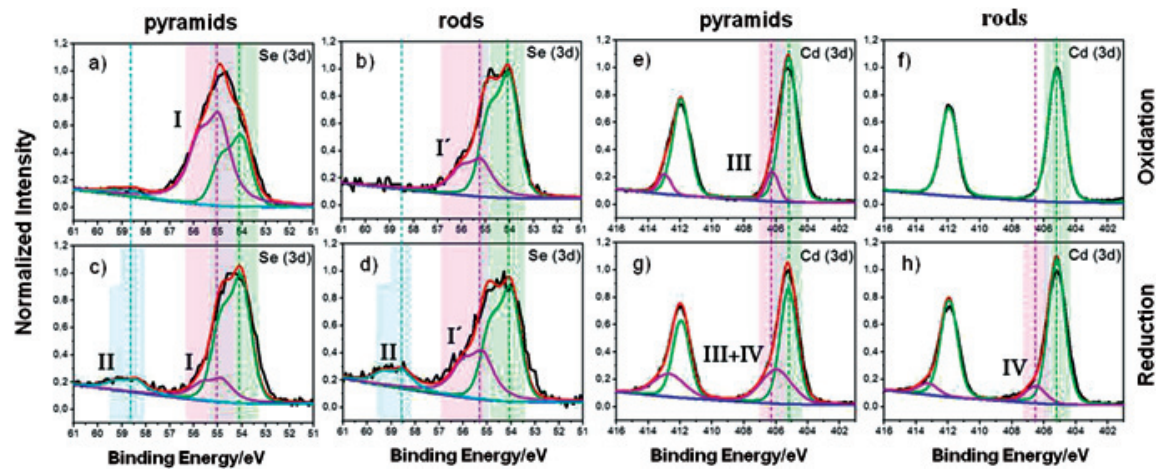

Figure 7: XPS spectra of Se and Cd 3d regions of pyramidal shaped and rod-like CdSe NCs, after first oxidation (a, e and b, f, respectively) and further reduction (c, g and d, h). The labeled components I and $I^{\prime}$ are products generated upon electrochemical oxidation which remain stable in rod-like NCs ( $I^{\prime}$ in b compared to $I^{\prime}$ in d) but can be partially reduced in a further cathodic scan in the case of pyramidal NCs (I in a compared to I in C). Component II corresponds to oxidation products in air (see text). III corresponds to Cd oxidation products obtained upon electrochemical oxidation occurring exclusively in pyramidal NCs but not in rod-like ones. IV corresponds to the reduction of $\mathrm{Cd}^{2+}$ to $\mathrm{Cd}^{0}$ upon electrochemical cathodic scan and further oxidation to $\mathrm{CdO}$ in air.

rod-like and pyramidal NCs have been identified. The first row in Figure 7 corresponds to Se and Cd spectra obtained upon the application of an anodic scan from 0 to $+2 \mathrm{~V}$ according to the previous voltammograms shown in Figure 6. New oxidized components can be found in the XPS Se spectra: component I at a binding energy of $55.0 \mathrm{eV}$ in Figure 7a for pyramids, and I' at a binding energy of $55.4 \mathrm{eV}$ in Figure $7 \mathrm{~b}$ for rods. Both components appear at higher binding energies than those components corresponding to non-oxidized CdSe NCs (green spectra). The intensity of the oxidized component is larger for pyramids than for rods, indicating that the oxidation proceeds faster in pyramids. In other words, the oxidation is less effective for Se sites in rods than in pyramids, what highlights that the presence of chloride capping the NCs surface must also have an influence on the redox reactions of Se sites. In order to further characterize differences produced in the samples during the anodic process, the potential was subsequently scanned in the cathodic direction (spectra in Figures $7 \mathrm{c}$ and $7 \mathrm{~d}$, second row). For a further reduction treatment, the previously oxidized Se sites in the rods (component $I^{\prime}$ ) remain constant both in binding energy position and intensity demonstrating that the anodic oxidation of the rod-like CdSe NCs capped with ODPA and without $\mathrm{Cl}$ is irreversible. However, the previously oxidized Se component for pyramids clearly shows a partial reversibility, with a drastic decrease in intensity (component I in Figure $7 \mathrm{a}$ vs component I in Figure 7c). This suggests that the oxidation mech- 
anism for the Cl-capped pyramidal NCs must be essentially different compared to the one taking place for rod-like NCs, involving the formation of a Se product which does not result in the oxidation of rod-like NCs. The reversibility in the pyramids is attributed to the formation of Se-Cl species in the presence of $\mathrm{Cl}$ [45].

On the other hand, pyramids also show an oxidation process taking place at the $\mathrm{Cd}$ sites (Figure 7e). As it can be clearly observed, a new contribution at higher binding energies relative to the $\mathrm{Cd} 3 \mathrm{~d} 5 / 2$ and $\mathrm{Cd} 3 \mathrm{~d} 3 / 2$ peaks is recorded for pyramidal NCs (component III in Figure 7e). Since the main difference between rods and pyramids when it comes to the chemical properties of the surface, is the presence of chloride anions attached to $\mathrm{Cd}$ sites, we conclude that the appearance of this extra contribution must arise from such $\mathrm{Cd}$ sites indicating its oxidation. In rods, however, the $\mathrm{Cd}$ sites remain unchanged. The binding energy position of the new $\mathrm{Cd}$ contribution is in good agreement with the formation of more oxidized $\mathrm{Cd}$ species. In any event this component does not appear in samples that have not been electrochemically oxidized. In the present study the voltammetric response of rods and pyramids differ mainly in the peak recorded at $1 \mathrm{~V}$ (see voltammogram in Figure 6) suggesting that the new Cd contribution can indeed be considered as a fingerprint of the oxidation of the $\mathrm{Cd}$ sites capped with $\mathrm{Cl}$. For $\mathrm{Cd}$ sites in rods the reduction process (Figure $7 \mathrm{~h}$ ) yields a higher binding energy component (component IV). Similarly to the effect observed for Se sites (component II in Figures 7c and 7d), since the sample has been reduced during this step (cathodic scan), these peaks can only be the result of unavoidable environmental oxidation when the samples are transferred from the electrochemical cell to the XPS chamber. In the case of $\mathrm{Cd}$ sites, the oxidation must proceeds from $\mathrm{Cd}^{2+}$ previously reduced to $\mathrm{Cd}^{0}$ in the cathodic scan. This effect is also observed for pyramids, where the new component must be added to the previous one obtained upon the anodic scan in the presence of chloride (sum of components III + IV). Thus, while oxidation at the Se sites occurs both for pyramids and rods, the oxidation at the $\mathrm{Cd}$ sites takes place only in pyramids. Comparing with the CV, the only oxidation process that takes place exclusively in pyramids corresponds to the peak at $1 \mathrm{~V}$, which we thus attribute to the oxidation of the $\mathrm{Cd}-\mathrm{Cl}$ sites. In conclusion, XPS ascertains that applying an anodic scan produces irreversible corrosion of Se in rods, but certain reversibility in pyramidal NCs capped with chloride ligands. The combination of CV and XPS allows for the identification of different $\mathrm{Cd}$ and Se environments and for testing the stability of CdSe NCs under different electrochemical treatments. The combination of these techniques may be very useful to understand the response of NCs in devices where an active NCs layer works under bias $[16,46]$. 


\section{Interaction with carbon $\mathrm{sp}^{2}$ surfaces}

The incorporation of chloride modifies the original ligand shell, reducing the density of long-chain organic ligands, thus allowing a closer contact at the interface between NCs and graphitic surfaces, as sketched in Figure 8. This was firstly observed in 2007, when including carbon nanotubes dispersed in DCE during the synthesis of CdSe rods, pyramidal NCs attached to the nanotubes were obtained $[7,33]$. Since then, several works have evidenced the possibility to decorate any carbon $\mathrm{sp}^{2}$ lattice (also other wurtzite NCs types such as $\mathrm{ZnO}$ ) [47] following the same procedure, which guaranties a monolayer of NCs on the graphene surface $[29,33]$. As explained before, the original shell combining ODPA ${ }^{2-}$ and anhydrides for CdSe is modified by the presence of chloride anions, which displace the anhydrides selectively and promotes the interaction with C-sp ${ }^{2}$ surfaces. This incorporation of chloride strengthens the interaction, as demonstrated by the fact that NCs with larger amounts of $\mathrm{Cl}$ on their surface (determined by XPS) decorate the C-sp ${ }^{2}$ surfaces more efficiently than particles with a lower amount [29]. The decoration of different surfaces following the same procedure can be seen in Figure 9, where microscopy images show different carbon $\mathrm{sp}^{2}$ systems decorated with CdSe NCs: single wall carbon nanotubes (Figure 10a), multiwall carbon nanotubes (Figure 10b), the surface of HOPG (Figure 10c) and flakes peeled off from the former substrate (Figure 10d). It is worth mentioning that, in contrast to the labile interaction of NCs monolayers covered with long alkyl chains obtained by means of techniques such as Langmuir-Blodgett, the monolayers obtained by this method (named pot-deposition) [29] are relatively strong and do not detach from the carbon $\mathrm{sp}^{2}$ lattice by washing the substrates in toluene or under mild sonication conditions.

High Angle Annular Dark Field (HAADF) Scanning Transmission Electron Microscopy (STEM) tomography studies were performed to ascertain the three di-

a)

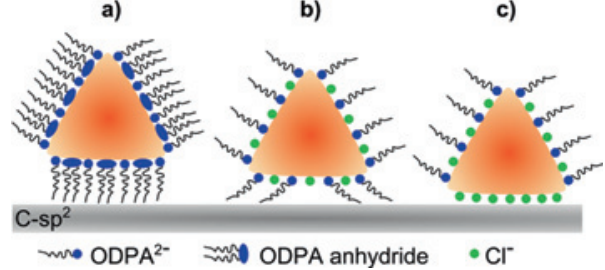

Figure 8: Scheme depicting the incorporation of chloride (green dots) in the original ODPA-related ligand shell and the interaction with carbon $\mathrm{sp}^{2}$ surfaces. (a) The original shell combines ODPA ${ }^{2}$ - and anhydrides; (b) the presence of chloride displaces the anhydrides selectively and (c) promotes the interaction with C-sp ${ }^{2}$ surfaces. 

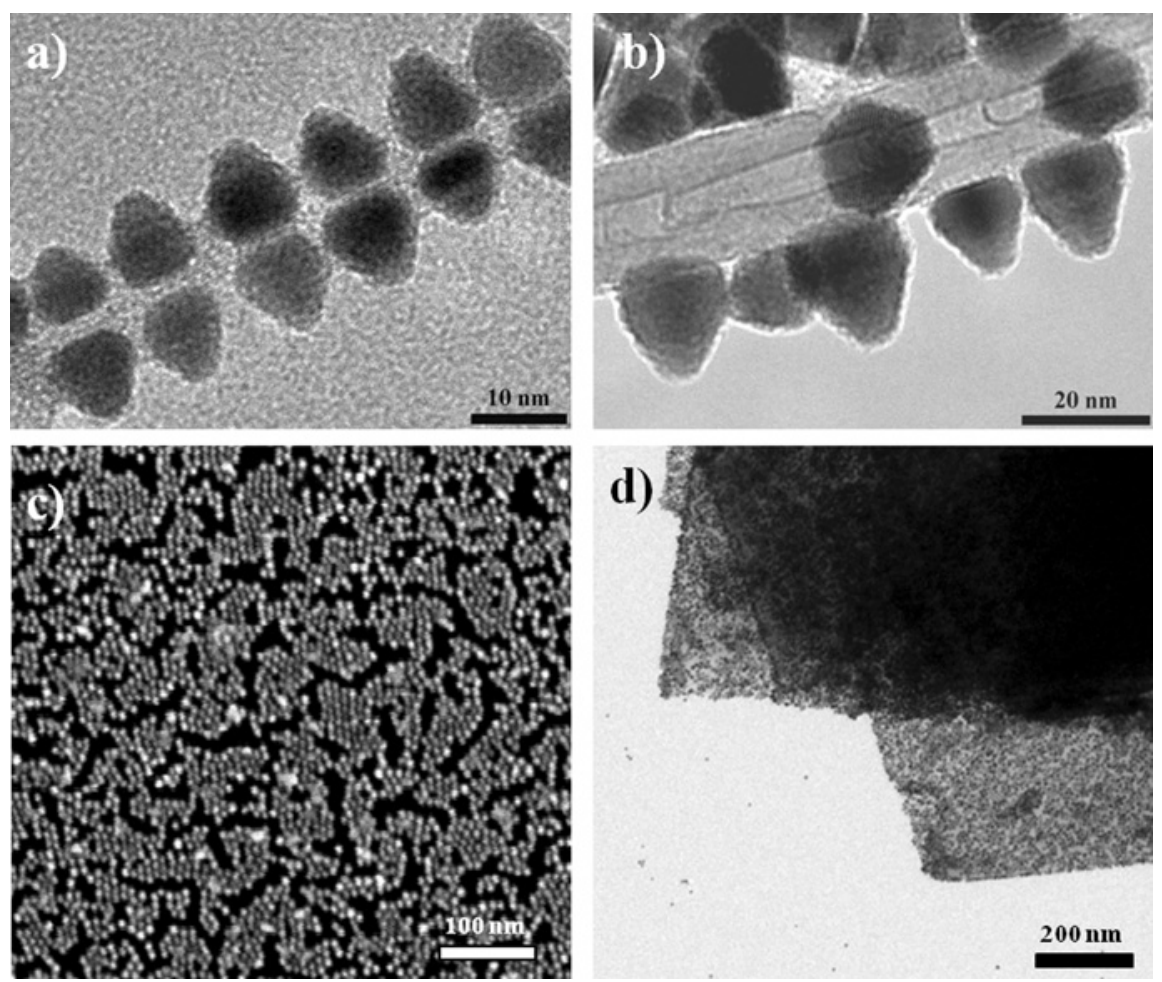

Figure 9: Electron microscopy images showing different carbon $\mathrm{sp}^{2}$ systems decorated with CdSe NCs: a) single wall, b) multiwall carbon nanotubes, c) the surface of a substrate of HOPG (highly oriented pyrolytic graphite) and peeled off flakes.

mensional geometry of the attached NCs. These studies revealed that the NCs are hexagonal-based with the (0001) planes attached the outer graphene layer. The shape of the base of the pyramids attached to the carbon nanotube is very close to a regular hexagon but slightly distorted in most of the particles, what can be explained as a consequence of the present amount of chloride anions available during the reshaping process [32]. As discussed in [30] the orientation of the NCs is slightly tilted respect the tube axis, which could be related with the chiral vector of the outer wall of the nanotube. In Figure 10 a change in the angle of the tilt between NCs 1 and 2 and NCs 3 to 5 can be seen. This might be explained by a change in the chirality of the nanotube wall possibly related to the bonding of CdSe NCs to both sides of the nanotubes. 


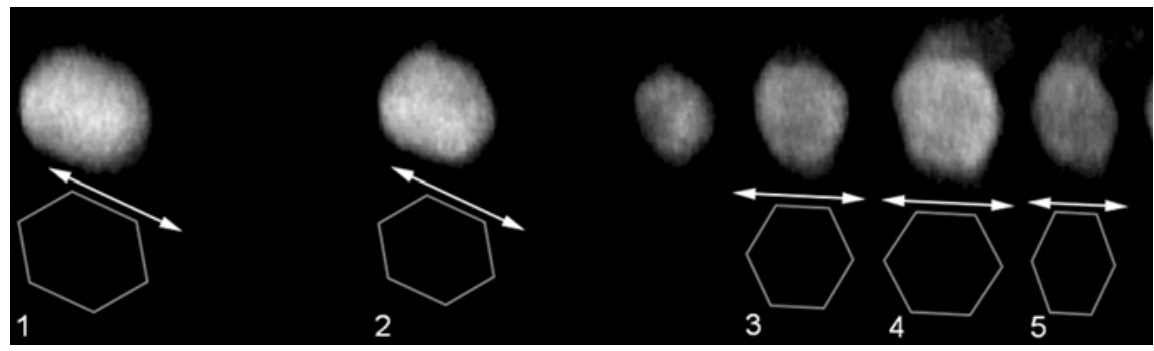

Figure 10: Tomography HAADF-STEM image showing the hexagonal shape of the base of the pyramids on a carbon nanotube, together with a change in the orientation between NCs 1 and 2 and 3 to 5 .

\section{The influence of chlorine on the synthesis of PbS NCs}

As mentioned in the introduction, several NCs syntheses are influenced by chlorine-containing compounds. Contrary to the shape modification and attachment to graphitic surfaces observed for wurtzite CdSe NCs, the synthesis of rocksalt $\mathrm{PbS}$ NCs yields different structures. In this case, chlorine-containing cosolvents, such as DCE, 1,2-dichloropropane (DCP), or 1,1,2-trichloroethane (TCE) alter nucleation and growth rates during the first stages of NCs formation, where Ostwald ripening plays a negligible role. The presence of chloride triggers the oriented attachment of NCs into ultrathin single-crystal sheets with dimensions on the micrometer scale, as it can be seen in Figure 11. X-ray scattering (SAXS) characterization ascertained distances between stacked layers corresponding to an interplanar crystalline oleic acid bilayer [14]. Since NCs try to minimize the most energetically unfavorable surface facets by fast growth perpendicular to the respective facet, relatively large PbS NCs (larger than $10 \mathrm{~nm}$ ) typically shows the shape of truncated cubes with mainly $\{100\}$ surface facets. The $\{111\}$ facets and the most reactive ones, $\{110\}$, are usually visible in smaller NCs [48]. In the case of small PbS NCs (up to $\sim 3 \mathrm{~nm}$ ) they should still exhibit the reactive $\{110\}$ facets through which oriented attachment takes place. The stacked structures is driven by dense packing of oleic acid ligands on $\{100\}$ facets, preventing bulk crystal growth. The chemical procedure involves the reaction between lead oleate and thioacetamide dissolved in dimethylformamide and TOP at $100{ }^{\circ} \mathrm{C}$ [49]. For CdSe NCs it was ascertained that chloride anions are released from the mixture of TOP and DCE (Figure 4). In the case of PbS NCs, since also TOP is used as solvent for the sulphur source, the influence of DCE might also release chloride anions, although the reaction temperature is much lower $\left(100^{\circ} \mathrm{C}\right)$. Additionally, in this case, the presence 

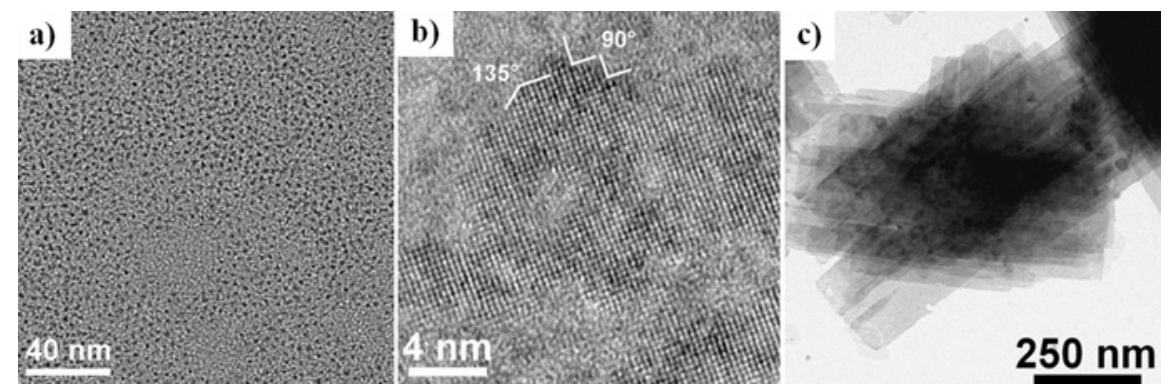

Figure 11: Structural evolution of $\mathrm{PbS}$ dots that, in the presence of the chlorinated co-solvent TCE, evolve into 2D sheets by oriented attachment (b) and stacked (c) with distances between layers corresponding to an interplanar crystalline oleic acid bilayer.

of acetate anions may also play a role in the final NCs shape and may also compete for different crystallographic facets during the evolution of the reaction, which is the topic of on-going investigations, including the use of these sheets as transistors [50].

\section{Summary}

A brief overview of the influence of haloalkanes mainly on the synthesis of wurtzite CdSe NCs by hot injection has been presented. According to XPS studies the halides get incorporated into the CdSe ligand shell. In the case of chloride anions, their incorporation modifies the shape of CdSe nanorods into pyramids. The reshaping is triggered by the reaction between TOP and the halogen-containing compound, releasing halide anions that alter the nucleation, first stages of growth or the ripening regime, depending on their chemical structure. In the case of chloride, their amount incorporated into the ligand shell and the corresponding NCs shape can be controlled by properly tuning the $\mathrm{Cd} / \mathrm{Se}$ precursor molar ratio. According to NMR and XPS, the chloride incorporation proceeds through selective displacement of anhydrides of octadecylphosphonic acid. The incorporation of chloride modifies not only the chemical environment of the ligand shell and the shape but also the redox response of the NCs. XPS ascertains that applying an anodic scan produces irreversible corrosion of Se in rods, but partial reversibility in pyramidal NCs capped with chloride ligands. The shape modification and change in the ligand shell promotes the NCs attachment to carbon $\mathrm{sp}^{2}$ lattices, such as those of carbon nanotubes, highly oriented pyrolytic graphite or graphene. This methodology guaranties in one pot a monolayer of NCs on the sur- 
face, what opens room for hybrid systems including semiconductor NCs-graphene for photovoltaics, catalysts, or energy storage. Finally, it has been shown that the influence of haloalkanes on the synthesis of rock-salt PbS NCs triggers oriented attachment, rendering them into 2D ultrathin sheets with micrometer dimensions. These sheets can be very advantageous favoring electrical transport usually blocked by long ligands in NCs-based devices.

Acknowledgement: The author thanks the Spanish Ministry of Economy and Competitiveness through MAT-2009-13488 and the Comunidad de Madrid through PHAMA_2.0 S2013/MIT-2740.

\section{References}

1. C. B. Murray, D. J. Norris, and M. G. Bawendi, J. Am. Chem. Soc., 115 (1993) 8706.

2. R. Li, J. Lee, B. Yang, D. N. Horspool, M. Aindow, and F. Papadimitrakopoulos, J. Am. Chem. Soc. 127 (2005) 2524.

3. S. J. Lim, W. Kim, S. Jung, J. Seo, and S. K. Shin, Chem. Mater. 23 (2011) 5029.

4. M. R. Kim, K. Miszta, M. Povia, R. Brescia, S. Christodoulou, M. Prato, S. Marras, and S. L. Manna, ACS Nano 6 (2012) 11088.

5. J. Lim, W. K. Bae, K. U. Park, L. zur Borg, R. Zentel, S. Lee, and K. Char, Chem. Mater. 25 (2013) 1443.

6. M. Meyns, N. G. Bastus, Y. Cai, A. Kornowski, B. H. Juarez, H. Weller, and C. Klinke, J. Mater. Chem. 20 (2010) 10602.

7. B. H. Juarez, C. Klinke, A. Kornowski, and H. Weller, Nano Lett. 7 (2007) 3564.

8. P. S. Nair, K. P. Fritz, and G. D. Scholes, Small 3 (2007) 481.

9. J. Joo, H. B. Na, T.Yu, J. H. Yu, Y. W. Kim, F. Wu, J. Zhang, and T. Hyeon, J. Am. Chem. Soc. 125 (2003) 11100.

10. K. An, N. Lee, J. Park, S. C. Kim, Y. Hwang, J. G. Park, J. Y. Kim, J. H. Park, M. J. Han, J. Yu, and T. Hyeon, J. Am. Chem. Soc. 128 (2006) 9753.

11. M. Saruyama, M. Kanehara, and T. Teranishi, J. Am. Chem. Soc. 132 (2010) 3280.

12. R. L. Penn and J. F. Banfield, Science 281 (1998) 969.

13. J. F. Banfield, S. A. Welch, H. Zhang, T. T. Ebert, and R. L. Penn, Science 289 (2000) 751.

14. C. Schliehe, B. H. Juarez, M. Pelletier, S. Jander, D. Greshnykh, M. Nagel, A. Meyer, S. Foerster, A. Kornowski, C. Klinke, and H. Weller, Science 329 (2010) 550.

15. A. H. Ip, S. M. Thon, S. Hoogland, O. Voznyy, D. Zhitomirsky, R. Debnath, L. Levina, L. R. Rollny, G. H. Carey, A. Fischer, K. W. Kemp, I. J. Kramer, Z. Ning, A. J. Labeller, K. W. Chou, A. Amassian, and E. H. Sargent, Nat. Nanotechnol. 7 (2012) 577.

16. J. Tang, K. W. Kemp, S. Hoogland, K. S. Jeong, H. Liu, L. Levina, M. Furukawa, X. Wang, R. Debnath, D. Cha, K. W. Chou, A. Fischer, A. Amassian, J. B. Asbury, and E. H. Sargent, Nat. Mater. 10 (2011) 765.

17. J. S. Owen, J. Park, P. E. Trudeau, and A. P. Alivisatos, J. Am. Chem. Soc. 130 (2008) 12279.

18. N. C. Anderson and J. S. Owen, Chem. Mater. 25 (2013) 69. 
19. W. K. Bae, J. Joo, L. A. Padilha, J. Won, D. C. Lee, Q. Lin, W. K. Koh, H. Luo, V. I. Klimov, and J. M. Pietryga, J. Am. Chem. Soc. 134 (2012) 20160.

20. B. I. MacDonald, A. Martucci, S. Rubanov, S. E. Watkins, P. Mulvaney, and J. J. Jasieniak, ACS Nano 6 (2012) 5995.

21. L. Qu and X. G. Peng, J. Am. Chem. Soc. 124 (2002) 2049.

22. P. V. Kamat, J. Phys. Chem. C 112 (2008) 18737.

23. C. Palencia, K. Lauwaet, L. de la Cueva, M. Acebroìn, M. Meyns, C. Klinke, J. M. Gallego, R. Otero, and B. H. Juarez, Nanoscale 6 (2014) 6812.

24. H. Liu, J. S. Owen, and A. P. Alivisatos, J. Am. Chem. Soc. 129 (2007) 305.

25. R. Gomes, A. Hassinen, A. Szczygiel, Q. Zhao, A. Vantomme, J. C. Martins, and Z. Hens, J. Phys. Chem. Lett. 2 (2011) 145.

26. A. J. Morris-Cohen, M. D. Donakowski, K. Knowles, and E. Weiss, J. Phys. Chem. C 114 (2010) 897.

27. J. T. Kopping and T. E. Patten, J. Am. Chem. Soc. 130 (2008) 5689.

28. G. Ohms, G. Grossmann, and B. Schwab, Sulfur Silicon Relat. 68 (1992) 77.

29. F. Iacono, C. Palencia, L. de la Cueva, M. Meyns, L. Terracciano, A. Vollmer, M. J. de la Mata, C. Klinke, J. M. Gallego, B. H. Juarez, and R. Otero, ACS Nano 7 (2013) 2559.

30. A. B. Hungría, B. H. Juarez, C. Klinke, H. Weller, and P. A. Midgley, Nano Res. 1 (2008) 89.

31. R. S. Rogers, Tetrahedron Lett. 33 (1992) 7473.

32. M. Meyns, F. Iacono, C. Palencia, J. Geweke, M. D. Coderch, U. E. A. Fittschen, J. M. Gallego, R. Otero, B. H. Juarez, and C. Klinke, Chem. Mater. 26 (2014) 1813.

33. B. H. Juarez, Meyns, A. Chanaewa, Y. Cai, C. Klinke, and H. Weller, J. Am. Chem. Soc. 130 (2008) 15282.

34. P. Hoyer and H. Weller, J. Phys. Chem., 99 (1995) 14096.

35. L. Kavan, T. Stoto, M. Gratzel, D. Fitzmaurice, and V. Shklover, J. Phys. Chem. 97 (1993) 9493.

36. F. Cao, G. Oskam, P. C. Searson, J. M. Stipkala, T. A. Heimer, F. Garzad, and G. Meyer, J. Phys. Chem. 99 (1995) 11974.

37. C. Wang, M. Shim, P. Guyot-Sionnest, Science 291 (2001) 2390.

38. S. K. Haram, B. M. Quinn, and A. J. Bard, J. Am. Chem. Soc. 123 (2001) 8860.

39. E. Kucur, J. Riegler, G. A. Urban, and T. Nann, J. Chem. Phys. 119 (2003) 2333.

40. L. de la Cueva, K. Lauwaet, R. Otero, J. M. Gallego, C. Alonso, Band . H. Juarez, J. Phys. Chem. C 118 (2014) 4998.

41. A. J. Bard and M. S. Wrighton, J. Electrochem. Soc. 124 (1977) 1706.

42. J. Jasieniak and P. Mulvaney, J. Am. Chem. Soc. 129 (2007) 2841.

43. J. H. Engel, Y. Surendranath, and A. P. Alivisatos, J. Am. Chem. Soc. 134 (2012) 13200.

44. S. K. Poznyak, N. P. Osipovich, A. Shavel, D. V. Talapin, N. Gao, A. Eychmueller, and N. Gaponik, J. Phys. Chem. B 109 (2005) 1094.

45. M. Lamoureux and J. Milne, Polyhedron 9 (1990) 589.

46. D. V. Talapin and C. B. Murray, Science 310 (2005) 86.

47. A. Chanaewa, B. H. Juarez, H. Weller, and C. Klinke, Nanoscale 4 (2012) 251.

48. K. S. Cho, D. V. Talapin, W. Gaschler, and C. B. Murray, J. Am. Chem. Soc. 127 (2005) 7140.

49. M. Nagel, G. S. Hickey, A. Frömsdorf, A. Kornowski, and H. Weller, Z. Phys. Chem. 221 (2007) 427.

50. S. Dogan, T. Bielewicz, Y. X. Cai, and C. Klinke, App. Phys. Lett. 101 (2012) 073102. 